\title{
Structures argumentales de prédicats $N$ Prép $N$. Emplois réciproques et non réciproques
}

\author{
Argument structures of $N$ Prep $N$ predicates. \\ Reciprocal and non reciprocal uses
}

\author{
Agnieszka K. Kaliska \\ Université Adam Mickiewicz, Poznań \\ agnie.kaliska@gmail.com
}

\begin{abstract}
The purpose of this article is to provide the lexical analysis of predicates such as face à face ('face to face') and côte à côte ('side by side'), which are generally reciprocal and symmetric. Some of them are also used in an unusual way, that is, the structures in which they appear do not meet the requirements set for symmetric constructions. This imbalance, as we will show, has for the origin a semantic difference between the arguments $x$ and $y$ which must belong to the same semantic class if we want them to be in a symmetric relation. The question of semantic similarity within the argument structures is thus of greater importance for the semantic completeness of the reciprocal predicates. The question will be for us to recognize if, when a semantic difference occurs, we would have to deal with different (non reciprocal) meanings and if so how to describe them.
\end{abstract}

Keywords: argument structure, reciprocity, symmetric relations, manner predicates

\section{INTRODUCTION}

L'objectif de la présente étude est d'analyser les emplois des prédicats tels que face à face, nez à nez, côte à côte, généralement réciproques et symétriques. Certains d'entre eux s'emploient aussi dans des constructions un peu étranges qui ne vérifient pas les règles que l'on utilise d'habitude pour bien identifier les relations 
symétriques. L'on démontrera que ce déséquilibre a pour origine une disconvenance sémantique entre les arguments $x$ et $y$ qui, pour qu'une relation de symétrie soit possible, doivent appartenir à la même classe sémantique. La question d'homologie sémantique des arguments est ainsi d'importance supérieure pour la complétude sémantique des prédicats étudiés. Le but de notre étude est de vérifier si, en cas de disconvenance sémantique, nous aurions à faire à des significations différentes et si oui comment les décrire.

Le français sera notre principale langue de référence. Ce n'est qu'en deuxième lieu que nous passerons au polonais et à l'italien pour compléter notre analyse et confirmer l'intuition selon laquelle le changement sémantique observable dans les exemples français a pour origine une capacité d'extension sémantique que l'on trouve également dans d'autres langues. Les mêmes outils seront donc utilisés pour analyser les exemples français et les exemples italiens et polonais.

Nous nous appuierons sur des exemples de corpus : certains (notés : Wiki) sont issus d'une copie de données de la Wikipédia et restent trouvables sur les sites de l'encyclopédie ; d'autres (notés : Internet) ont été tirés de documents accessibles via les moteurs de recherche Google et Google Books. La liste des adresses sources se trouve à la fin de l'article.

\section{SYMÉTRIE ET RÉCIPROCITÉ}

Les verbes comme se marier et coïncider sont intrinsèquement symétriques parce que leurs emplois vérifient un ensemble de règles transformationnelles ayant pour origine le raisonnement mathématique faisant appel à des règles qui permettaient de constater que deux figures géométriques étaient en relation de symétrie l'une avec l'autre. Nous expliquons ces règles dans la sous-partie qui suit.

\subsection{Propriétés transformationnelles des prédicats symétriques}

En mathématique, une relation est dite symétrique si elle vérifie la règle : $x R y>y R x$. Autrement dit: "une relation $R$ est une relation symétrique si chaque fois que le couple $(x, y)$ vérifie la relation $R$, alors le couple $(y, x)$ vérifie aussi la relation $R$ » (Pasero, Sabatier, Stéfanini, 2010, p. 18 ; voir aussi : Winter, 2018, p. 2). Les arguments $x$ et $y$ du prédicat symétrique jouent donc le même rôle l'un vis-à-vis de l'autre. En syntaxe, cela se manifeste par l'interchangeabilité des deux sujets coordonnés ou par l'interchangeabilité du sujet et du complément dans les constructions non paratactiques. Par exemple, si $x$ et $y$ effectuent l'action $a$, on peut en déduire qu'au même moment $y$ et $x$ effectuent la même action $a$; de même si $x$ effectue l'action $a$ avec $y$, en même temps $y$ effectue la même action $a$ avec $x$. Soulignons quand même qu'il ne s'agit pas dans le cas du complément introduit par 
avec d'un simple commitatif. L'accompagnement aurait souvent un caractère adventice (ce que symbolise l' $E$ non asterisqué ci-dessous) contrairement au rôle qu'assume dans l'exécution de l'action l'argument $y$, élément requis (essentiel) du prédicat intrinsèquement symétrique (ce que symbolise l' $E$ astérisqué). Par exemple :

(1) Max se promène dans le parc (avec Nina $\mid \mathrm{E})$.

(2) Nina se promène dans le parc (avec Max |E).

(3) Max s'est marié (avec Nina $\mid$ *E).

(4) Nina s'est mariée (avec Max| *E).

- si les $N$ introduits par avec ne sont pas les compléments essentiels du prédicat se promener, ils sont les compléments essentiels du prédicat intrinsèquement symétrique, en l'occurrence se marier. Certains compléments essentiels peuvent, certes, être omis dans ce qu'on appelle le discours, la phrase Max s'est marié étant parfaitement acceptable mais la différence entre Nina se promène dans le parc et Max s'est marié est cruciale : dans Nina se promène dans le parc, le pronom se est réflexif; dans Max s'est marié, le pronom se n'est jamais réflexif (i.e. Max ne s'est pas marié avec lui-même, il s'est marié avec quelqu'un qui n'a pas été précisé).

Dans l'article consacré aux verbes symétriques français, Borillo (1971, p. 18) souligne que les permutations illustrées par le schéma $x R y>y R x$ doivent être «non significatives », autrement dit le verbe est symétrique si le sens reste le même quel que soit l'ordre des arguments $x$ et $y$. Chez Nkollo (2009) une troisième condition permettant d'identifier les prédicats symétriques apparaît - c'est la possibilité de référer aux arguments $x$ et $y$ par le nom employé au pluriel en position initiale, ce qui remet en cause la distinction faite entre le sujet-agent, d'un côté, et l'objet-patient, de l'autre, pour désigner par ces deux étiquettes $x$ et $y$, arguments du prédicat symétrique employés dans une construction non paratactique (voir la notion désignée par le terme kompresja chez Nkollo, 2009, p. 178-179). Cette condition confirme que les prédicats symétriques tels que se marier sont réciproques («[S]ymmetric predicates exhibit plain reciprocity », Winter, 2018, p. 3). Les manipulations syntaxiques exercées sur l'exemple suivant permettent de vérifier la pertinence des règles esquissées ci-dessus :

(5) En octobre 2000 André Golota se retrouva au tapis face à face avec Mike Tyson.

Le prédicat face à face accepte la permutation des arguments $x$ et $y$, sujets coordonnés, ainsi que la permutation des mêmes arguments employés comme sujet et complément dans les constructions non paratactiques :

(6) En octobre 2000 Mike Tyson se retrouva au tapis face à face avec André Golota.

(7) André Golota et Mike Tyson se retrouvèrent face à face au tapis en octobre 2000.

(8) Mike Tyson et André Golota se retrouvèrent face à face au tapis en octobre 2000. 
L'exemple vérifie également la règle de compression de deux arguments à l'aide de l'étiquette boxeurs faisant appel aux aspects tels que métier exercé par deux hommes et dont la référence sera ensuite confirmée par le contexte :

(9) Les boxeurs se retrouvèrent face à face au tapis en octobre 2000.

Le marqueur temporel en octobre 2000 diminue, parait-il, le risque d'ambiguïté lié au fait que l'interprétation selon laquelle il y a eu deux face-à-face auxquels ont participé les boxeurs, chacun de son côté, tandis que leurs rivaux n'ont pas été précisés, n'est pas impossible (voir l'interprétation de l'exemple avec cohabiter dans : Pasero, Sabatier, Stéfanini, 2010, p. 119).

\subsection{Perspective fonctionnelle de la phrase et (a)symétrie}

Un paradoxe émerge lors de l'analyse fonctionnelle des phrases (5) - (8) car si leurs prédicats sont symétriques, une certaine asymétrie se laisse néanmoins remarquer si l'on admet que dans une construction non paratactique on a à faire à une situation dans laquelle quelqu'un qui se retrouve face à face avec quelqu'un d'autre se voit en quelque sorte obligé de lui faire face comme si celui-ci constituait pour lui une menace ou un défi. Ainsi serait-il juste de distinguer entre symétrie et réciprocité, quoique les opinions sur la juste interprétation de deux notions soient différentes parmi les auteurs (p.ex. Dimitriadis, 2008; Winter, 2018). Il faut souligner néanmoins qu'une mise en relief de l'argument sujet n'invalide pas les propriétés sémantico-lexicales du prédicat. Ainsi une certaine disproportion qui émerge lorsqu'on analyse un exemple comme :

(10) En octobre 2000 André Golota se retrouva au tapis face à face avec Mike Tyson, champion du monde poids lourd

n'invalide pas les propriétés symétriques du prédicat face à face puisque la permutation est toujours possible :

(11) En octobre 2000, Mike Tyson, champion du monde poids lourd, se retrouva au tapis face à face avec André Golota.

Ce qui change alors c'est la perspective fonctionnelle de la phrase, dite aussi la perspective thème-rhème, et non pas les propriétés lexico-grammaticales du prédicat auxquelles fait d'ailleurs référence la sémantique vériconditionnelle (s'il est vrai que $x$ se retrouva au tapis face à face avec $y$, alors il est vrai qu'au même moment $y$ se retrouva au tapis face à face avec $x$ ). L'asymétrie thème-rhème des constructions ayant pour prédicat les noms de relations symétriques illustre au fait l'analogie apparente mais trompeuse de deux types d'analyse que sont, d'un côté, la séman- 
tique lexicale, vériconditionnelle, et, de l'autre, l'approche fonctionnelle, fortement discursive (Kaliska, 2018, p. 97). Si la thématisation est d'abord un procédé stylistique et que l'analyse thème-rhème est basée sur l'énoncé tel quel, la prédication est une notion sémantique, abstraite et, par la suite, indépendante des configurations hic et nunc, à chaque fois différentes, que l'on rencontre dans le discours.

\section{RÔLE DU VERBE}

Les verbes tels que se retrouver (voir les exemples de 5 à 11), être et tomber (voir plus loin) auxquels s'ajoutent les prédicats $N$ Prép $N$ analysés assument un rôle connu mais souvent évincé de support - grammatical, aspectuel et stylistique (Daladier, 1998 ; Vetulani, 2000 ; Kaliska, 2018). Les verbes supports ne sont donc pas prédicatifs. Dans les cas analysés, les structures argumentales sont d'ailleurs fortement contraintes et elles dépendent des prédicats $N$ Prép $N$, ce qui vient d'être signalé dans le $\S 2.1$ et sera dévelopé dans la suite de notre étude. Le rôle prédicatif est par contre porté par l'ensemble de la structure $V N$ Prép $N$ dans le cas des constructions complexes telles que travailler $N$ Prép $N$ (p.ex. travailler la main dans la main) et marcher $N$ Prép $N$ (p.ex. marcher la main dans la main) où travailler et marcher sont sémantiquement pleins et ont l'impact sur les propriétés sélectionnelles de l'ensemble (voir, p.ex. : les exemples numérotés [1] dans le tableau 3, $\S 4.1)$.

\section{HOMOLOGIE SÉMANTIQUE DES ARGUMENTS $X$ ET $Y$. EMPLOIS RÉCIPROQUES}

Dans son étude consacrée aux verbes symétriques polonais Nkollo (2009, p. 41) souligne qu'à l'origine des propriétés syntaxiques définitoires pour la classe des prédicats symétriques il y a l'homologie sémantique des arguments $x$ et $y$. Autrement dit, il est nécessaire qu'on puisse attribuer aux arguments $x$ et $y$ le même trait sémantique. L'analyse des exemples relevés sur corpus confirme qu'il faut entendre par cela l'appartenance des arguments à la même classe sémantique. M. Gross (1981, p. 21) le signala en disant que deux $N$ d'une relation de symétrie doivent être « sémantiquement voisins ».

L'analyse des exemples français sera menée en trois étapes. Nous commencerons par les emplois réciproques à structures argumentales homogènes simples, telles que [+ humain] \& [+ humain], [+ animal] \& [+ animal], [+ inanimé] \& [+ inanimé]. Nous nous pencherons ensuite sur des emplois à structures argumentales mixtes, telles que [+ humain] \& [+ animal] - nous prouverons qu'il s'agit dans leurs cas des emplois réciproques. Ce n'est qu'après que nous passerons à l'analyse des cas de disconvenance sémantique, non symétriques et non réciproques. 


\subsection{Structures argumentales homogènes}

Un premier échantillon d'exemples se trouve dans le tableau 1. Nous y avons réunis des prédicats qui sélectionnent en principe des arguments humains, le trait [+ humain] faisant appel aux noms de personnes, de collectivités et, par métonymie, aux noms d'institutions :

Tableau 1. Prédicats réciproques aux arguments $x$ et $y$ humains

\begin{tabular}{|c|c|c|c|}
\hline \multirow[t]{2}{*}{ Prédicat } & \multicolumn{3}{|c|}{$\begin{array}{l}\text { [+ humain] } \& \text { [+ humain] } \\
\text { où comme humains sont employés les noms ou syntagmes désignant les : }\end{array}$} \\
\hline & personnes & collectivités & institutions \\
\hline au coude-à-coude & $\begin{array}{l}\text { Les sondages nationaux } \\
\text { montrent alors une pro- } \\
\text { gression constante du séna- } \\
\text { teur du Vermont, désormais } \\
\text { au coude à coude avec son } \\
\text { opposante }[. . .] \text {. (Wiki) }\end{array}$ & $\begin{array}{l}\text { Les Templiers reviennent } \\
\text { peu à peu dans le match, } \\
\text { dans le dernier quart-temps, } \\
\text { ils sont au coude à coude } \\
\text { avec les Black Panthers } \\
\text { [...]. (Wiki) }\end{array}$ & $\begin{array}{l}{[\mathrm{L}] \text { e Parti national }(N P) \text { se }} \\
\text { retrouve au coude à coude } \\
\text { avec le Congrès national } \\
\text { africain }[. . .] . \text { (Wiki) }\end{array}$ \\
\hline la main dans la main & $\begin{array}{l}\text { En fait, Lamanon et l'abbé } \\
\text { Mongez, tous deux natura- } \\
\text { listes et minéralogistes, tra- } \\
\text { vaillèrent la main dans la } \\
\text { main. (Wiki) }\end{array}$ & $\begin{array}{l}{[\ldots] \text { Wallons et Flamands }} \\
\text { marchent en ce moment-là } \\
\text { main dans la main }[. . .] . \\
\text { (Wiki) }\end{array}$ & $\begin{array}{l}{[\mathrm{L}] \text { 'église travaillera main }} \\
\text { dans la main avec les au- } \\
\text { torités coloniales }[\ldots] . \\
\text { (Wiki) }\end{array}$ \\
\hline face à face & $\begin{array}{l}\text { Toki se retrouva alors face } \\
\text { à face avec le sinistre } \\
\text { homme de science. (Wiki) }\end{array}$ & $\begin{array}{l}{[\mathrm{L}] \text { e } 4 \text { décembre } 1676, \text { les }} \\
\text { deux armées se retrouvèrent } \\
\text { face à face juste au nord de } \\
\text { Lund. (Wiki) }\end{array}$ & $\begin{array}{l}\text { Après avoir signé des } \\
\text { accords avec l'Iran, l'URSS } \\
\text { tombe face à face avec les } \\
\text { États-Unis en tentant } \\
\text { d'acquérir les provinces } \\
\text { turques d'Ardahan et Kars. } \\
\text { (Wiki) }\end{array}$ \\
\hline
\end{tabular}

À cela s'ajoutent quelques exceptions car, d'après l'usage, un face-à-face peut se tenir également entre l'homme et Dieu, p. ex. :

(12) Si le face à face avec Dieu est le rapport essentiel, sans Dieu, tout est solitude. (Malègue, voir : TLFi, article : face)

(13) Moïse parle face à face avec Dieu comme un ami parle à son ami. (Bible, Exode, 33, p. 11)

- et il s'agit en l'occurrence d'une relation de symétrie ce que confirme l'emploi :

(14) Dieu parle face à face avec Mö̈se. (Internet)

Fait partie de cette classe la variante littéraire regard à regard qui n'accepte pas les emplois métonymiques avec les noms d'institutions : 
(15) Les voici tous les deux [en parlant de deux garçons], face à face, regard à regard, bien campés sur leurs jambes, mains en avant à la façon des judokas, prêts pour un premier assaut. (J. Dissler, Internet)

Les prédicats à possibilités d'emploi plus larges se trouvent dans le tableau 2. En effet, nez à nez et côte à côte s'emploient facilement non seulement avec les arguments humains mais aussi avec les noms d'animaux, voire avec les inanimés. Voilà quelques exemples :

Tableau 2. Prédicats réciproques aux arguments $x$ et $y$ animés et inanimés

\begin{tabular}{|l|l|l|l|}
\hline Prédicat & [+ humain] \& [+ humain] & [+ animal] \& [+ animal] & [- animé] \& [- animé] \\
\hline nez à nez & $\begin{array}{l}\text { Dans l'appartement, Fabien } \\
\text { se retrouve nez à nez avec } \\
\text { Gérard, le mari d'Anne. } \\
\text { (Wiki) }\end{array}$ & $\begin{array}{l}\text { La souris sort de son trou et } \\
\text { se retrouve nez à nez avec } \\
\text { un chat. (notre exemple) }\end{array}$ & $\begin{array}{l}\text { La Renault de Max est garée } \\
\text { nez à nez avec le cabriolet } \\
\text { de sa femme. (notre exem- } \\
\text { ple) }\end{array}$ \\
\hline côte à côte & $\begin{array}{l}\text { Churchill et Clementine sont } \\
\text { placés côte à côte et enta- } \\
\text { ment bientôt une histoire } \\
\text { d'amour qui durera toute } \\
\text { leur vie. (Wiki) }\end{array}$ & $\begin{array}{l}\text { Les deux oiseaux marchent } \\
\text { côte à côte avec la tête en } \\
\text { arrière touchant presque le } \\
\text { dos. (Wiki) }\end{array}$ & $\begin{array}{l}\text { [...] pour que les ouvres } \\
\text { russes se trouvent côte à côte } \\
\text { avec les cuvres françaises. } \\
\text { (Wiki) }\end{array}$ \\
\hline
\end{tabular}

Or, la nature sémantique des arguments détermine l'interprétation du prédicat. Il apparaît que celle-ci change dans le cas du prédicat nez à nez selon qu'on l'emploie avec des arguments humains, animaux ou objets inanimés. Ainsi, nez à nez qui signifie grosso modo 'l'un en face de l'autre' véhicule une nuance de soudaineté et de surprise en parlant des humains et animaux mais il n'a pas la même nuance quand on parle des objets inanimés.

Il faudrait proposer un type d'analyse pareil aux configurations d'arguments des prédicats la main dans la main et côte à côte (voir ci-dessous) quand ils sont employés dans des emplois métaphoriques où ils signifient 'harmonieusement' ou 'en accord' en parlant soit des humains qui collaborent soit des idées abstraites qui coexistent. Il est à souligner qu'ici encore le verbe et les arguments déterminent la juste interprétation de deux prédicats car ceux-ci risquent d'avoir un sens différent si leurs arguments sont humains et si le verbe qui les précède est un verbe de déplacement autre que marcher. Le prédicat la main dans la main signifie alors 'en se tenant par la main', tandis que côte à côte veut dire 'l'un à côté de l'autre'. Ces emplois seraient, historiquement, les premiers ([1] - voir le tableau 3), les autres ([2]) sont leurs extensions car, si le verbe est marcher et qu'il est employé en parlant des humains, il n'est pas toujours évident ce que font ceux qui marchent côte à côte ou la main dans la main. Font-ils des pas de marche l'un à côté de l'autre, en se tenant par la main, ou vivent-ils en plein accord? En effet, les emplois figurés des 
unités marcher, côte à côte et la main dans la main se sont élargis au point de rendre difficile le choix de la juste interprétation sans recourir aux autres éléments de la phrase. Voilà quelques exemples :

Tableau 3. Prédicats réciproques la main dans la main et côte à côte aux arguments $x$ et $y$ humains, d'un côté, et humains ou abstraits, de l'autre

\begin{tabular}{|c|c|c|}
\hline Prédicat & [+ humain] \& [+ humain] & [+ abstrait] \& [+ abstrait $]$ \\
\hline $\begin{array}{l}\text { la main dans la } \\
\text { main }[1]\end{array}$ & $\begin{array}{l}\text { Le couple sort de la clinique, main dans la } \\
\text { main [...]. (Wiki) Denny lui dit qu'elle est } \\
\text { magnifique et ils partent ensemble la main } \\
\text { dans la main. (Wiki) Très émue Solenne se } \\
\text { contente de l'enlacer puis de l'embrasser. } \\
\text { Ils marchent la main dans la main dans les } \\
\text { rues de La Rochelle. (T. Slimani, Internet) }\end{array}$ & \\
\hline $\begin{array}{l}\text { la main dans la } \\
\text { main [2] }\end{array}$ & $\begin{array}{l}\text { En fait, Lamanon et l'abbé Mongez, tous } \\
\text { deux naturalistes et minéralogistes, } \\
\text { travaillèrent la main dans la main. (Wiki) }\end{array}$ & $\begin{array}{l}\text { Mais l'histoire et le mythe marchent } \\
\text { souvent main dans la main en Écosse. } \\
\text { (Wiki) }\end{array}$ \\
\hline côte à côte [1] & $\begin{array}{l}\text { Au matin, descendus sans se concerter } \\
\text { [...] ils se promènent côte à côte, timides } \\
\text { [...]. (A. Lascar, Internet) Tandis qu'ils } \\
\text { marchent côte à côte, il ose lui prendre la } \\
\text { main. La jeune fille y est sensible. } \\
\text { (M. Bruna, Internet) }\end{array}$ & \\
\hline côte à côte [2] & $\begin{array}{l}\text { L'amour, c'est marcher avec l'autre côte } \\
\text { à côte, corps à corps, cour à cour. (Inter- } \\
\text { net) }\end{array}$ & $\begin{array}{l}\text { L'importation des esclaves et les progrès } \\
\text { du christianisme dans la population mar- } \\
\text { chent côte à côte. (J. Cooper, Internet) }\end{array}$ \\
\hline
\end{tabular}

Notons aussi qu'il existe des emplois où deux prédicats, la main dans la main et côte à côte, sont employés :

(16) $[\mathrm{O}] n$ dirait deux amants qui marchent côte à côte et la main dans la main. (A. Dumas, Internet)

\subsection{Structures argumentales mixtes}

$\mathrm{Au}$ cours de l'analyse, nous avons pu noter que certaines configurations d'arguments se laissent réunir pour former des classes plus larges telles que les êtres vivants. Il en est ainsi, par exemple, pour les structures d'arguments mixtes, [+ humain] \& [+ animal], opposées aux structures d'arguments homogènes telles que [+ humain] \& [+ humain] et [+ animal] \& [+ animal] que nous avons pu observer plus haut (voir le tableau 2 dans le $\S 4.1$ ). Par exemple :

(17) [...] Indiana [Jones] se retrouve nez à nez avec un cobra [...]. (Wiki) 
Voilà d'autres emplois à structures d'arguments mixtes :

(18) [...] M. Dupuis tombera nez à nez avec la vache [...]. (Wiki)

(19) Yakouba s'enfonce dans la jungle et se retrouve nez à nez avec un lion... (Internet)

(20) Qu'advient-il lorsqu'un homme se retrouve nez à nez avec un ours? (Internet)

(21) Pyrénées : un randonneur se retrouve nez à nez avec une ourse. (Internet)

(22) Bousculé en pleine plongée, il [c'est-à-dire : un plongeur] se retrouve nez à nez avec un requin blanc. (Internet)

Il est également possible qu'un des arguments réfère aux êtres fantastiques tandis que l'autre est un nom d'animal :

(23) [...] la princesse Lliane et les jeunes hauts-elfes vont chasser. Alors qu'ils attaquent une harde de cerfs, ils tombent nez à nez avec un groupe de loups géants. (Wiki)

Or, comme en témoigne le tableau 2, le prédicat nez à nez s'emploie déjà dans des structures assez variées où les $x$ et $y$ partagent tous les deux les traits sémantiques définitoires soit pour les humains, soit pour les animaux, soit encore pour les inanimés. Il est donc naturel de se poser la question si dans le cas des emplois mixtes cités ci-dessus nous aurions à faire aux mêmes prédicats symétriques et comment définir alors la classe sémantique des arguments $x$ et $y$.

Les permutations permettant de dire qu'il s'agit dans les cas cités des constructions symétriques sont possibles. Il faut par contre prendre en considération qu'avec un changement de perspective certaines modifications peuvent être indispensables pour assurer l'acceptabilité de l'exemple. Ainsi, par exemple, un cobra deviendra plutôt le cobra et sera ensuite dévelopée par une relative, p. ex. le cobra que $P$. Toujours est-il que la mise en position initiale d'un nom d'animal est possible :

(24) Le cobra qui vient d'attaquer le jeune homme se retrouva nez à nez avec Indiana.

(25) Malheureusement pour elle, la vache tombera nez à nez avec M. Dupuis.

Les manipulations pareilles sont également acceptables dans le cas des sujets coordonnés :

(26) M. Dupuis et la vache tomberont nez à nez l'un avec l'autre, ce qui finira mal pour l'animal.

(27) Malheureusement pour la vache, celle-ci et M. Dupuis tomberont nez à nez l'un avec l'autre.

Il est vrai, pourtant, que les exemples dans lesquels les noms d'humains apparaissent d'un côté et d'autre du verbe sont les plus fréquents. Ceux-ci se prêtent plus facilement aux manipulations syntaxiques comme celles essayées ci-dessus. Cela n'a 
probablement qu'un fondement purement anthropocentrique car, en effet, la mise en position initiale d'un nom d'animal irait de pair, semble-t-il, avec une personnification de celui-ci. On fait ainsi de l'animal un personnage réel qui est doté d'une volonté, agit et est doué de sentiment (voir, p.ex. malheureusement pour elle [i.e. pour la vache]). Dans l'usage, les noms d'animaux en position initiale sont pratiquement introuvables en français (d'après la recherche faite sur Internet), mais il paraît qu'ils ne sont pas impossibles :

(28) Tout d'un coup, l'animal se retrouva nez à nez avec un chasseur. (notre exemple)

Notons finalement qu'une configuration d'arguments mixte est également possible dans le cas du prédicat face à face :

(29) Julie [...] est tombée face à face avec un ours polaire. (Internet)

Jusqu'à présent nous n'avons vu que des emplois symétriques avec une possibilité de la mise en relief qui, certes, introduit une certaine asymétrie sur le plan fonctionnel de la phrase mais n'invalide pas les propriétés lexico-grammaticales des prédicats réciproques. Dans la suite de notre étude nous nous pencherons sur des emplois en quelque sorte atypiques pour lesquels aucune des propriétés de symétrie n'est vérifiée.

\section{DISCONVENANCE SÉMANTIQUE ENTRE LES ARGUMENTS $X$ ET $Y$. EMPLOIS NON RÉCIPROQUES}

Nous proposons de parler des prédicats non symétriques lorsqu'il est impossible d'employer un nom par lequel on pourrait référer à la fois à l'argument $x$ et à l'argument $y$. Comparons l'exemple pour lequel un tel remplacement est possible avec l'exemple où une pareille substitution ne peut se faire :

(30) Dans l'appartement, Fabien se retrouve nez à nez avec Gérard, le mari d'Anne. (Wiki) vs. (30a) Dans l'appartement, deux hommes se retrouvent nez à nez.

- où deux hommes renvoie à deux arguments humains (Fabien et Gérard) ;

(31) Dans le cimetière attenant à la mine, Marty tombe nez à nez avec la tombe du Doc. (Wiki)

vs. (31a) *Dans le cimetière attenant à la mine ils tombent nez à nez l'un avec l'autre.

- où le pronom sujet ils ne peut renvoyer à la fois à l'argument humain (Marty), d'un côté, et l'argument inanimé (la tombe) de l'autre. 
La disconvenance sémantique entre les arguments $x$ et $y$ entraîne également le blocage des permutations que vérifient normalement les prédicats symétriques. Par exemple :

(32) Dans le cimetière..., Marty tombe nez à nez avec la tombe du Doc. (Wiki) vs. (32a) *Dans le cimetière..., la tombe du Doc tombe nez à nez avec Marty. vs. (32b) *Dans le cimetière..., Marty et la tombe du Doc tombent nez à nez... vs. (32c) *Dans le cimetière..., la tombe du Doc et Marty tombent nez à nez...

On peut se demander ce qui détermine dans l'exemple (31) l'emploi de la construction tomber sur $N$ qui, à la différence du verbe pronominal se retrouver employé plus haut, semble asymétrique et non réciproque (il n'est pas vrai que si $x$ tombe sur $y, y$ tombe sur $x$ au même moment). Le verbe tomber revient d'ailleurs dans un autre exemple de même nature :

(33) Il [c'est-à-dire : le héros d'un roman] grimpe en haut d'une très grande tour en ruines du château et tombe nez à nez avec une grille. (Wiki)

vs. (33a) *La grille tombe nez à nez avec le héros...

vs. (33b) *Lui et la grille tombent nez à nez, l'un avec l'autre.

vs. (33c) *La grille et le héros tombent nez à nez, l'un avec l'autre.

vs. (33d) *Ils [c'est-à-dire : le héros et la grille] tombent nez à nez, l'un avec l'autre.

Or, nous observons les mêmes blocages là où sont employés le verbe pronominal se retrouver et le verbe neutre être; quant aux arguments l'un est humain (p.ex. les artistes et les vétérans), tandis que l'autre est une idée abstraite (p.ex. les problèmes labyrinthiques liés à la délivrance difficile de visas... et la famine) :

(34) [L]es artistes qui se sont retrouvés, de la même façon, nez-à-nez avec les problèmes labyrinthiques liés à la délivrance difficile de visas aux Etats-Unis [...]. (Internet)

vs. (34a) *Les problèmes liés à la délivrance des visas se sont retrouvés nez à nez avec les artistes.

vs. (34b) *Les artistes et les problèmes liés à la délivrance des visas se sont retrouvés nez à nez.

vs. (34c) *Les problèmes liés à la délivrance des visas et les artistes se sont retrouvés nez à nez.

vs. (34d) *Ils [c'est-à-dire : les artistes et les problèmes liés à la délivrance des visas] se sont retrouvés nez à nez.

(35) Les vétérans de l'armée du « Bonus » [...] sont face à face avec la famine. (Internet) vs. (35a) *La famine est face à face avec les vétérans de l'armée.

vs. (35b) *Les vétérans de l'armée et la famine sont face à face.

vs. (35c) * La famine et les vétérans de l'armée sont face à face.

vs. (35d) *Ils [c'est-à-dire : les vétérans de l'armée et la famine] sont face à face.

La disconvenance sémantique entre $x$ et $y$ est donc un phénomène indépendant du choix du verbe support (tomber, se retrouver ou être). Les exemples de même nature sont multiples : 
(36) Certes, dans ces pays, on se retrouve nez à nez avec la prostitution des adultes et des enfants, la déforestation massive, la pêche à la dynamite, les faux orphelinats, la maltraitance des femmes et des enfants, le travail des enfants... (Internet)

(37) Nez à nez avec la misère, la cruauté et la débauche parisienne, c'est en tant qu'espion du lieutenant-général de police qu'il couvrira sa première affaire. (Internet)

Dans certains cas, la compression est pourtant envisageable puisqu'un abstrait comme mort se laisse parfois personnifier. Il faut par contre que la totalité de la phrase s'y adapte. Par exemple :

(38) Ainsi, se retrouvent-ils face à face, l'Homme et la mort. (notre exemple)

S'il est clair que les exemples cités sont tous corrects (sauf, bien évidemment, les résultats biaisés de leurs transformations), leur sens, semble-t-il, ne peut être le même que celui des exemples analysés plus haut, à structures d'arguments homogènes. Ici, plus qu'ailleurs, on fait référence à des situations dans lesquelles celui qui se retrouve face à face avec quelque chose se voit en quelque sorte obligé de se mesurer à quelque chose de difficile, l'inverse n'étant pas vrai. Or, de tels emplois sont fréquents chez les grands auteurs qui déplorent certains états d'âme et tristes expériences que subit l'Homme :

(39) Je me regardais, si je puis dire, moi tout seul, j'étais face à face avec ma solitude. (Malègue, TLFi, article : face)

(40) [...] Je sentais la rougeur me monter au visage [...] c'était la première fois qu'on me mettait ainsi face à face avec mon ignorance. (Dumas, TLFi, article : face)

Il en découle alors que parmi les emplois dits réciproques certains ne satisfont pas aux règles de symétrie. Dans les cas que nous venons d'analyser, les états que subissent les $x$ et les états que subissent les $y$ sont loin d'être équivalents.

\section{TABLEAU RÉCAPITULATIF DES EMPLOIS FRANÇAIS. PREMIÈRES CONCLUSIONS}

La caractérisation de la classe des prédicats symétriques à l'aide des critères syntaxiques nous a permis de délimiter les emplois que l'on peut qualifier comme réciproques. Ces critères étaient, rappelons-les, l'interchangeabilité des arguments $x$ et $y$ (c'est-à-dire de deux sujets coordonnées ou du sujet et du complément dans des constructions non paratactiques), la possibilité de référer aux arguments $x$ et $y$ par le nom employé au pluriel en position initiale. Or, il s'est avéré que parmi les configurations d'arguments qui vérifient les règles de symétrie présentées, il y en a qui sont parfaitement homogènes (p.ex. [+ humain] \& [+ humain]), d'un côté, et 
mixtes (p.ex. [+ humain] \& [+ animal]), de l'autre. Un tel déséquilibre ne doit pas forcément être conflictuel du point de vue de l'actualisation du sens car ce dernier reste conservé même dans les emplois où un argument est humain et l'autre est un animal. Il serait par contre nécessaire de distinguer entre, d'un côté, les emplois réciproques à structures argumentales mixtes et, de l'autre, les emplois non réciproques où la disconvenance entre les $x$ et $y$ est beaucoup plus importante (p.ex. [+ humain] \& [-animé] ou [+ humain] \& [+ abstrait]), puisqu'elle déclenche le blocage immédiat de toutes les manipulations syntaxiques.

Les différences observables sur le plan syntaxique sont également présentes sur le plan sémantique. Nous proposons de différencier alors entre les formules telles que 'l'un en face de l'autre' et 'le premier en face du second' pour décrire de cette façon les emplois réciproques d'un côté et les emplois non réciproques de l'autre.

Les résultats de notre analyse ont été réunis dans le tableau 4. Les exemples ont été regroupés selon le prédicat, le sens et quatre classes sémantiques que nous avons différenciées pour caractériser les structures argumentales dans les emplois réciproques, à savoir :

(I) les humains, y compris les collectivités et institutions dont les membres sont les humains (p.ex. pour le prédicat au coude-à-coude) - à distinguer d'une classe plus large, notamment :

(II) les êtres vivants, c'est-à-dire les humains, animaux, êtres fantastiques et divinités, y compris Dieu (p.ex. pour le prédicat nez à nez) - à distinguer d'une classe encore plus large, c'est-à-dire :

(III) les objets concrets, c'est-à-dire perceptibles par les sens, y compris les objets animés (animaux et humains) et les objets inanimés (p.ex. pour le prédicat côte à côte) ;

(IV) les notions abstraites (p.ex. pour le prédicat la main dans la main).

La classe des non restreints, que l'on différencie par exemple en lexique-grammaire pour dire qu'un nom qui entre dans une structure n'est spécifié d'aucune manière (Nnr - voir, p.ex. : Gross, 1975), ne trouve pas sa place dans l'analyse des prédicats étudiés.

Tableau 4. Tableau récapitulatif des emplois réciproques et non réciproques des prédicats regard à regard, au coude-à-coude, côte à côte, face à face, la main dans la main et nez à nez

\begin{tabular}{|l|l|l|l|c|}
\hline \multicolumn{1}{|c|}{ Prédicat } & \multicolumn{1}{|c|}{ Sens et nuance } & $\begin{array}{c}\text { Structure } \\
\text { argumentale }\end{array}$ & $\begin{array}{c}\text { Arguments } \boldsymbol{x} \text { et } \boldsymbol{y} \text { - exemples tirés } \\
\text { des emplois étudiés }\end{array}$ & $\boldsymbol{x} \boldsymbol{R} \boldsymbol{y}>\boldsymbol{y} \boldsymbol{R}$ \\
\hline $\begin{array}{l}\text { REGARD } \\
\text { Á REGARD }\end{array}$ & $\begin{array}{l}\text { 'l'un en face de l'autre en se } \\
\text { regardant droit dans les yeux' }\end{array}$ & $\begin{array}{l}{[+ \text { humain }]} \\
\&[+ \text { humain }]\end{array}$ & deux garçons & + \\
\hline $\begin{array}{l}\text { AU COUDE- } \mathrm{A}- \\
\text { COUDE }\end{array}$ & 'en plein accord' & $\begin{array}{l}{[+ \text { humain }]} \\
\&[+ \text { humain }]\end{array}$ & $\begin{array}{l}\text { le sénateur du Vermont et son oppo- } \\
\text { sante } ; \text { les Templiers \& les Black } \\
\text { Panther }\end{array}$ & + \\
\hline
\end{tabular}




\begin{tabular}{|c|c|c|c|c|}
\hline Prédicat & Sens et nuance & $\begin{array}{c}\text { Structure } \\
\text { argumentale }\end{array}$ & $\begin{array}{c}\text { Arguments } x \text { et } y \text { - exemples tirés } \\
\text { des emplois étudiés }\end{array}$ & $x R y>y R$ \\
\hline \multirow[t]{3}{*}{ CÔTE À CÔTE } & 'l'un à côté de l'autre' & $\begin{array}{l}{[+ \text { concret }]} \\
\&[+ \text { concret }]\end{array}$ & $\begin{array}{l}\text { Churchill et Clementine; deux } \\
\text { oiseaux; les oeuvres russes et les } \\
\text { oeuvres françaises }\end{array}$ & + \\
\hline & \multirow[t]{2}{*}{$\begin{array}{l}\text { 'en plein accord ou } \\
\text { harmonieusement' }\end{array}$} & $\begin{array}{l}\text { [+ humain] } \\
\&[+ \text { humain }]\end{array}$ & l'homme et la femme & + \\
\hline & & $\begin{array}{l}\text { [+ abstrait }] \\
\&[+ \text { abstrait }]\end{array}$ & $\begin{array}{l}\text { l'importation des esclaves et le pro- } \\
\text { grès du christianisme }\end{array}$ & + \\
\hline \multirow[t]{2}{*}{ FACE À FACE } & $\begin{array}{l}\text { 'l'un en face de l'autre (avec } \\
\text { une nuance de surprise, de défi } \\
\text { ou avec une nuance d'intimité)' }\end{array}$ & $\begin{array}{l}{[+ \text { animé }]} \\
\&[+ \text { animé }]\end{array}$ & $\begin{array}{l}\text { Toki et le sinistre homme de science; } \\
\text { l'URSS et les États Unis ; Julie et } \\
\text { un ours polaire; l'homme et Dieu }\end{array}$ & + \\
\hline & $\begin{array}{l}\text { 'le premier en face du second } \\
\text { (avec une nuance de défi)' }\end{array}$ & $\begin{array}{l}\text { [+ humain }] \\
\&[+ \text { abstrait }]\end{array}$ & $\begin{array}{l}\text { les vétérans et la famine; l'homme } \\
\text { et la solitude; l'homme et son } \\
\text { ignorance }\end{array}$ & - \\
\hline \multirow{3}{*}{$\begin{array}{l}\text { LA MAIN } \\
\text { DANS } \\
\text { LA MAIN }\end{array}$} & 'en se tenant par la main' & $\begin{array}{l}\text { [+ humain }] \\
\&[+ \text { humain }]\end{array}$ & Danny et la fille & + \\
\hline & \multirow[t]{2}{*}{$\begin{array}{l}\text { 'en plein accord ou } \\
\text { harmonieusement' }\end{array}$} & $\begin{array}{l}{[+ \text { humain }]} \\
\&[+ \text { humain }]\end{array}$ & $\begin{array}{l}\text { la manon et l'abbé Mongez; } \\
\text { Wallons et Flamands; l'église et } \\
\text { les autorités coloniales }\end{array}$ & + \\
\hline & & $\begin{array}{l}\text { [+ abstrait }] \\
\&[+ \text { abstrait }] \\
\end{array}$ & l'histoire et le mythe & + \\
\hline \multirow[t]{4}{*}{ NEZ À NEZ } & $\begin{array}{l}\text { 'l'un en face de l'autre (avec une } \\
\text { nuance de surprise et de défi)' }\end{array}$ & $\begin{array}{l}\text { [+ animé }] \\
\&[+ \text { animé }]\end{array}$ & $\begin{array}{l}\text { Fabien et Gérard ; la souris et un } \\
\text { chat; Indiana et le cobra }\end{array}$ & + \\
\hline & $\begin{array}{l}\text { 'l'un en face de l'autre (sans la } \\
\text { nuance de surprise)' }\end{array}$ & $\begin{array}{l}{[- \text { animé }]} \\
\&[- \text { animé }]\end{array}$ & $\begin{array}{l}\text { la Renault de Max et le cabriolet } \\
\text { de sa femme }\end{array}$ & + \\
\hline & $\begin{array}{l}\text { 'le premier en face du second } \\
\text { (avec une nuance de surprise)' }\end{array}$ & $\begin{array}{l}\text { [+ humain }] \\
\&[- \text { animé }]\end{array}$ & $\begin{array}{l}\text { Marty et la tombe; l'homme et une } \\
\text { grille }\end{array}$ & - \\
\hline & $\begin{array}{l}\text { 'le premier en face du second } \\
\text { (avec une nuance de défi)' }\end{array}$ & $\begin{array}{l}\text { [+ humain }] \\
\&[+ \text { abstrait }]\end{array}$ & $\begin{array}{l}\text { les artistes et les problèmes liés } \\
\text { à la délivrance des visas }\end{array}$ & - \\
\hline
\end{tabular}

\section{PRÉDICATS N PREP N POLONAIS ET ITALIENS}

Certains équivalents des prédicats analysés issus d'autres langues manifestent des propriétés de polysémie analogues, ce qui permet de présumer qu'il s'agit en l'occurrence d'une faculté d'extension courante à l'origine de laquelle il se trouve le même concept, reproductible dans plusieurs langues.

Ci-dessous ont été réunies les paires d'emplois des prédicats équivalents, polonais et italiens : twarza $w$ twarz et faccia a faccia (fr. 'face à face'), nos w nos et naso a naso (fr. 'nez à nez'), oko w oko et occhi negli occhi (fr. 'les yeux dans les yeux'). Leurs structures argumentales sont variées : des plus simples et régulières (i.e. [+ humain] \& [+ humain]), par les configurations mixtes (i.e. [+ humain] \& [+ animal]), jusqu'aux emplois où $x$ et $y$ sont de nature différente (i.e. [+ humain] \& [+ abstrait]) et ne peuvent être permutés. Voilà quelques exemples : 
Tableau 5. Structures argumentales et exemples d'emploi des prédicats twarza w twarz, nos w nos, oko $w$ oko et faccia a faccia, naso a naso, occhi negli occhi

\begin{tabular}{|c|c|c|c|c|c|c|}
\hline \multirow{2}{*}{$\begin{array}{c}\text { Structure } \\
\text { argumentale }\end{array}$} & \multicolumn{6}{|c|}{ Exemples polonais et italiens } \\
\hline & $\begin{array}{c}\text { TWARZA } \\
\text { W TWARZ }\end{array}$ & $\begin{array}{l}\text { (A) FACCIA } \\
\text { A FACCIA }\end{array}$ & NOS W NOS & NASO A NASO & OKO W OKO & $\begin{array}{c}\text { OCCHI } \\
\text { NEGLI OCCHI }\end{array}$ \\
\hline $\begin{array}{l}\text { [+ humain }] \\
\&[+ \text { humain }]\end{array}$ & $\begin{array}{l}\text { Dulska, jak wi- } \\
\text { cher, przeleciała } \\
\text { salon i znalazła } \\
\text { sie nagle nos } \\
\text { w nos ze Zbysz- } \\
\text { kiem. (Internet) }\end{array}$ & $\begin{array}{l}\text { Katrin Creeve } \\
\text { era naso a naso } \\
\text { con un uomo } \\
\text { piuttosto alto. } \\
\text { (Internet) }\end{array}$ & $\begin{array}{l}\text { Cabaro zblizyt } \\
\text { się bardziej, aż } \\
\text { stanat nos w nos } \\
\text { z Brigganem. } \\
\text { (E. Schrefer, } \\
\text { Internet) }\end{array}$ & $\begin{array}{l}\text { Jame la strappò } \\
\text { via e si trovò } \\
\text { naso a naso con } \\
\text { il maestro. } \\
\text { (S. Pergameno, } \\
\text { Internet) }\end{array}$ & $\begin{array}{l}\text { W sequelu, które- } \\
\text { go akcja rozgry- } \\
\text { wa się w maju } \\
\text { 1936, Kwinto } \\
\text { znów staje oko } \\
\text { w oko z Krame- } \\
\text { rem [...]. (Wiki) }\end{array}$ & $\begin{array}{l}\mathrm{U}] \text { n uomo } \\
\text { (o una donna) si } \\
\text { trova occhi negli } \\
\text { occhi con la per- } \\
\text { sona che } \\
\text { vorrebbe [...]. } \\
\text { (Internet) } \\
\end{array}$ \\
\hline $\begin{array}{l}\text { [+ humain }] \\
\&[+ \text { animal }]\end{array}$ & $\begin{array}{l}{[\mathrm{N}] \text { ajmlodsi pod- }} \\
\text { różnicy [powinni] } \\
\text { stanać twarza } w \\
\text { twarz z tygrysem } \\
\text { w Loro Parku. } \\
\text { (Internet) }\end{array}$ & $\begin{array}{l}\text { Corsi verso la } \\
\text { riva per sfuggire } \\
\text { alla sua presa } \\
\text { mortale, ma li } \\
\text { mi trovai nuova- } \\
\text { mente faccia } \\
\text { a faccia con la } \\
\text { tigre. (V. Tatti, } \\
\text { Internet) }\end{array}$ & $\begin{array}{l}\text { Na nowym pla- } \\
\text { kacie [...] Bryce } \\
\text { Dallas Howard } \\
\text { stoi nos } \text { w nos } \\
\text { z dinozaurem, to- } \\
\text { czac z nim poje- } \\
\text { dynek na spoj- } \\
\text { rzenia. } \\
\text { (Internet) }\end{array}$ & \begin{tabular}{|l|} 
Si possono $[\ldots]$ \\
trovarsi quasi \\
naso a naso con \\
una tartaruga \\
gigante! \\
(Internet)
\end{tabular} & $\begin{array}{l}{[\mathrm{C}] z \text { lowiek styka }} \\
\text { się oko w oko ze } \\
\text { zwierzeciem } \\
{[\ldots] \text {. (Internet) }}\end{array}$ & $\begin{array}{l}\text { Li si scontra per } \\
\text { la prima volta, } \\
\text { occhi negli } \\
\text { occhi con il lupo } \\
\text { [...]. (Internet) }\end{array}$ \\
\hline $\begin{array}{l}{[+ \text { humain }]} \\
\&[+ \text { abstrait }]\end{array}$ & \begin{tabular}{|l|} 
[A]nnie sie uda- \\
to. Stanęta twa- \\
rza w twarz \\
z choroba, zmie- \\
rzyta sie z bólem \\
[...]. (M. Meeker, \\
Internet)
\end{tabular} & $\begin{array}{l}\text { Pietro si troverà } \\
\text { spalle al muro, } \\
\text { faccia a faccia } \\
\text { con la paura. } \\
\text { (Internet) }\end{array}$ & $\begin{array}{l}\text { W chwili tra- } \\
\text { gedii stanętam } \\
\text { oko w oko z inna } \\
\text { rzeczywistoś- } \\
\text { cia. (Internet) }\end{array}$ & - & $\begin{array}{l}\text { Stanat oko w oko } \\
\text { ze swoimi fobia- } \\
\text { mi. (M. Bylczyń- } \\
\text { ski, Internet) }\end{array}$ & $\begin{array}{l}\text { Solo chi si trova } \\
\text { occhi negli occhi } \\
\text { con la morte } \\
\text { e riesce a scon- } \\
\text { figgerla, com- } \\
\text { prende }[. . .] . \\
\text { (Internet) }\end{array}$ \\
\hline
\end{tabular}

Seuls les emplois où les arguments sont de même nature peuvent être traités comme des emplois réciproques. Ceux pour lesquels on ne peut trouver d'étiquette commune seraient donc des emplois inverses. Cette intuition est confirmée par une série de blocages morphosyntaxiques. Par exemple :

(41) Anna stanęła twarza w twarz z choroba, zmierzyła się z bólem.

vs. (41a) *Choroba stanęla twarza w twarz z Anna...

vs. (41b) *Anna i jej choroba stanęty twarza w twarz...

vs. (41c) *Choroba i Anna stanęty twarza w twarz...

vs. (41d) *One [c'est-à-dire : Anna i jej choroba] stanęty twarza w twarz...

(42) Pietro si troverà faccia a faccia con la paura.

vs. (42a) *La paura si troverà faccia a faccia con Pietro.

vs. (42b) *Pietro e la paura si troveranno faccia a faccia.

vs. (42c) * La paura et Pietro si troveranno faccia a faccia.

vs. (42d) *Si troveranno faccia a faccia [c'est-à-dire : Pietro e la paura].

En effet, les manipulations syntaxiques comme celles envisagées ci-dessus sont sans doute possibles dans le cas des emplois réguliers et ceux à configurations d'arguments mixtes. Par exemple : 
(43) Dulska znalazła się nos w nos ze Zbyszkiem.

vs. (43a) Zbyszek znalazt się nos w nos z Dulską.

(43) Katrin era naso a naso con il suo amico, Max.

vs. (43a) Max era naso a naso con la sua amica Katrin.

- pour les emplois réguliers ;

(44) Człowiek styka się nos w nos ze zwierzęciem.

vs. (44a) Zwierzę styka się nos w nos z człowiekiem.

(45) La ragazza si scontra occhi negli occhi con il lupo.

vs. (45a) Il lupo si scontra occhi negli occhi con la ragazza.

- pour les emplois mixtes.

Les nuances de surprise et de défi qui accompagnent les face-à-face, rencontres désignées par des prédicats comme nez à nez, nos w nos ou naso a naso, sont des éléments favorables à des extensions sémantiques comme celles illustrées plus haut. Il en est autrement pour l'accord et l'harmonie, états et qualités qui, comme leurs noms l'indiquent, n'acceptent pas de modifications aussi importantes au niveau de leurs structures argumentales. Ainsi, les prédicats ramię $w$ ramię, ręka $w$ rękę, d'un côté, et fianco a fianco, mano nella mano (ou, plus rarement, mano a mano), de l'autre, fonctionnent de la même manière que leurs équivalents français au coude-à-coude, côte à côte et la main dans la main. Voilà quelques exemples :

Tableau 6. Structures argumentales et exemples d'emploi des prédicats ramię w ramię, ręka w rękę et fianco a fianco, mano nella mano

\begin{tabular}{|c|c|c|c|c|}
\hline \multirow{2}{*}{$\begin{array}{c}\text { Structure } \\
\text { argumentale }\end{array}$} & \multicolumn{4}{|c|}{ Exemples polonais et italiens } \\
\hline & RAMIĘ W RAMIĘ & FIANCO A FIANCO & RĘKA W RĘKĘ & MANO NELLA MANO \\
\hline $\begin{array}{l}\text { [+ humain }] \\
\&[+ \text { humain }]\end{array}$ & $\begin{array}{l}\text { Nastolatkowie szli } \\
\text { ramie } \text { w ramie } \\
\text { z emerytami. } \\
\text { (Internet) }\end{array}$ & $\begin{array}{l}\text { Sally era fianco } \\
\text { a fianco con Estelle } \\
\text { nella nuovissima } \\
\text { cucina del suo nuo- } \\
\text { vissimo apparta- } \\
\text { mento. (E. Holly, } \\
\text { Internet) }\end{array}$ & $\begin{array}{l}\text { Dalej komendant } \\
\text { miasta, osiwiaty } \\
\text { wojennik ze staro- } \\
\text { zytnego pruskiego } \\
\text { rodu Ferberów, } \\
\text { szedt ręka w rękę } \\
\text { z burgrabia. (Deo- } \\
\text { tyma, Internet) }\end{array}$ & $\begin{array}{l}\text { Lele ed Elisa istinti- } \\
\text { vamente si ritrovano } \\
\text { mano nella mano } \\
\text { per la paura. } \\
\text { (Grimor, Internet) }\end{array}$ \\
\hline $\begin{array}{l}\text { [+ abstrait }] \\
\&[+ \text { abstrait }]\end{array}$ & $\begin{array}{l}\text { Innowacyjność } \text { i au- } \\
\text { tomatyzacja ida } \\
\text { ramię w ramię ze } \\
\text { wszystkim, co cyfro- } \\
\text { we } i \text { inteligentne } \\
\text { [...]. (Internet) }\end{array}$ & $\begin{array}{l}\text { Antico e moderno, } \\
\text { presente e passato } \\
\text { si intrecciano } \\
\text { e vanno fianco } \\
\text { a fianco. (Internet) }\end{array}$ & $\begin{array}{l}\text { Film, w którym } \\
\text { seks } i \text { humor ida } \\
\text { ręka } \text { w rękę }[. . .] \\
\text { (Internet) }\end{array}$ & $\begin{array}{l}{[\mathrm{L}] \text { e posizioni fon- }} \\
\text { damentaliste vanno } \\
\text { mano a mano con } \\
\text { atteggiamenti di na- } \\
\text { tura repressiva }[. . .] \\
\text { (Internet) }\end{array}$ \\
\hline
\end{tabular}


Nous avons donc à faire aux prédicats entièrement réciproques car mêmes les éventuelles différences entre $x$ et $y$ concernent l'homme et l'inanimé susceptible de personnification. En parlant par exemple d'une dépression ou d'une vie en guerre on utilise le prédicat côte à côte pour dire qu'on côtoie la mort: On vit mais, au fait, on marche côte à côte avec la mort (notre exemple), ce qui veut dire aussi que la mort nous côtoie (la phrase La mort nous côtoie étant acceptable).

\section{CONCLUSION}

Le sens des prédicats $N$ Prép $N$ réciproques a rarement une interprétation simple et unique. Certaines nuances dépendent strictement de la nature sémantique des arguments. On interprète autrement les emplois où les rôles sémantiques majeurs sont assumés par les êtres animés et ceux où les arguments $x$ et $y$ sont tous les deux inanimés ou abstraits. La réciprocité serait ainsi une notion complexe, recouvrant des aspects divers. À part quelques difficultés d'interprétation liées aux variations du sens dans les emplois réciproques, il paraît nécessaire de reconnaître qu'il existe également des extensions non réciproques parmi les emplois possibles des prédicats réciproques. Pour ces derniers, nous aurions donc à faire non pas à un prédicat mais à des prédicats dont les structures argumentales sont différentes et qui se laissent analyser à l'aide des tests syntaxiques permettant de confirmer ou de nier leur statut sémantique. Curieusement, la disconvenance sémantique entre les arguments $x$ et $y$ n'est pas un cas isolé qui soit limité aux emplois spécifiques des prédicats face à face et nez à nez en français. On l'observe également dans les emplois des prédicats polonais et italiens du même sens comme si, à leur origine, il y avait une sorte de moule réutilisable, appliqué à la même nuance de sens, notamment la nuance de défi. Or, le défi, étant de par sa nature unilatéral, génère inévitablement des emplois non réciproques. 


\section{BIBLIOGRAPHIE}

Borillo, A. (1971). Remarques sur les verbes symétriques français. Langue Française, 11. Paris : Larousse, 17-31, https://doi.org/10.3406/lfr.1971.5544.

Daladier, A. (1996). Le rôle des verbes support dans un système de conjugaison nominale et l'existence d'une voix nominale en français. Langages, 121. Paris : Larousse, 35-53, https://doi.org/10.3406/lgge.1996.1739.

Dimitriadis, A. (2008). Irreducible symmetry in reciprocal constructions. In : E. König, V. Gast (ed.), Reciprocals and reflexives: theoretical and cross-linguistic explorations. Berlin : De Gruyter Mouton, 375-410, https://doi.org/10.1515/9783110199147.375.

Gross, M. (1975). Méthodes en syntaxe. Paris : Hermann.

Gross, M. (1981). Les bases empiriques de la notion de prédicat sémantique. Langages, 63. Paris : Larousse, 7-52, https://doi.org/10.3406/lgge.1981.1875.

Kaliska, A.K. (2018). Wyktadniki instrumentalno-sposobowe a struktura semantyczna zdania. Polsko-francuska perspektywa porównawcza. Poznań : Adam Mickiewicz University Press.

Nkollo, M. (2009). Modele syntagmatyczne konstrukcji opartych na nazwach relacji symetrycznych. Poznań : Adam Mickiewicz University Press.

Pasero, R., Sabatier, P., Stéfanini, M.-H. (2010). Syntaxe et sémantique formelle des constructions verbales exprimant des relations symétriques. Langages, 179-180. Paris : Larousse, 115-141, https://doi.org/10.3917/lang.179.0115.

Vetulani, G. (2000). Rzeczowniki predykatywne języka polskiego. Poznań : Adam Mickiewicz University Press.

Winter, Y. (2018). Symmetric predicates and the semantics of reciprocal alternations. Semantics and Pragmatics, 11 (early access version). Cambridge : MIT, 1-48, http://dx.doi.org/10. 3765/sp.11.1.

\section{RESEAUGRAPHIE}

Trésor de la Langue Française informatisé, https://www.le-tresor-de-la-langue.fr/.

\section{CORPUS}

Wikipédia, l'encyclopédie libre (pour les exemples notés : Wiki), https://fr.wikipedia.org/wiki/ Wikip\%C3\%A9dia:Accueil_principal.

Exemples français notés Internet :

https://www.prixm.org/dieu-images-representation-ancien-testament/ [20 II 2019]

$\mathrm{https}$ //books.google.pl/books?id=hVgwBwAAQBAJ\&pg=PA45\&lpg=PA45\&dq=\%22deux+face-\%

C3\%A0-face $\% 22 \&$ source=bl\&ots=flWgt0P5k\&sig=ACfU3U3WAxzZHsF3KqEEBJlHiFbcGV

$9 \_$iw\&hl=pl\&sa=X\&ved=2ahUKEwjusf3TzMzgAhWqwosKHegdAFIQ6AEwDXoECAEQAQ $\# \mathrm{v}=$ onepage $\& \mathrm{q}=\% 22$ deux $\% 20$ face- $\% \mathrm{C3} \% \mathrm{~A} 0$-face $\% 22 \& \mathrm{f}=$ false [20 II 2019]

https://books.google.pl/books?id=NNPIEuVxqjcC\&pg=PA80\&lpg=PA80\&dq=\%22Tr\%C3\%A8s + $\% \mathrm{C} 3 \% \mathrm{~A} 9 \mathrm{mue}+$ Solenne $+\mathrm{se}+$ contente $+\mathrm{de}+1 \% \mathrm{E} 2 \% 80 \% 99$ enlacer + puis $+\mathrm{de}+1 \% \mathrm{E} 2 \% 80 \% 99 \mathrm{emb}$ rasser. + Ils + marchent + la + main + dans + la + main + dans + les + rues + de + La + Rochelle $\% 22 \&$ source

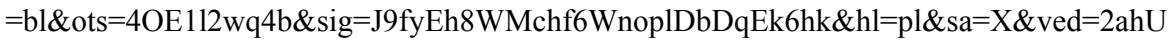
KEwjk8O6KyOLeAhUjBMAKHabKCZIQ6AEwAHoECAAQAQ\#v=onepage\&q=\%22Tr\% C3\%A8s\%20\%C3\%A9mue\%20Solenne\%20se\%20contente\%20de\%201\%E2\%80\%99enlacer \%20puis\%20de\%201\%E2\%80\%99embrasser.\%20Ils\%20marchent\%201a\%20main\%20dans\% 20la\%20main\%20dans\%20les\%20rues\%20de\%20La\%20Rochelle\%22\&f=false [20 XI 2018] 
https://books.google.pl/books?id=w92SO21XdlkC\&pg=PA151\&lpg=PA151\&dq=Au+matin, + des cendus + sans + se + concerter\&source $=$ bl\&ots $=$ LpqFnr6YNS\&sig $=$ ACfU3U1Ojpb2aAL8kfohn O9j2bpv3vowLA\&hl=pl\&sa=X\&ved=2ahUKEwi1-

KqztcvgAhXpposKHbAiB9kQ6AEwAHoECAAQAQ\#v=onepage\&q=Au\%20matin\%2C\%2 0descendus $\% 20$ sans $\% 20 \mathrm{se} \% 20$ concerter $\& \mathrm{f}=$ false [20 II 2019]

https://books.google.pl/books?id=w92SO21XdlkC\&pg=PA151\&dq=Au+matin, + descendus + sans + se + concerter $\& \mathrm{hl}=\mathrm{pl} \& \mathrm{sa}=\mathrm{X} \& \mathrm{ved}=0$ ahUKEwijuovCyOLeAhUFCSwKHWNpDDsQ6AEILT $\mathrm{AA \# v}=$ onepage $\& \mathrm{q}=\mathrm{Au} \% 20$ matin $\% 2 \mathrm{C} \% 20$ descendus $\% 20$ sans $\% 20 \mathrm{se} \% 20$ concerter $\& \mathrm{f}=$ false [20 XI 2018]

https://books.google.pl/books?id=oz86BgAAQBAJ\&pg=PA62\&dq=\%22Tandis + qu $\% E 2 \% 80 \% 99 \mathrm{i}$ ls + marchent $+\mathrm{c} \% \mathrm{C} 3 \% \mathrm{~B} 4 \mathrm{te}+\% \mathrm{C} 3 \% \mathrm{~A} 0+\mathrm{c} \% \mathrm{C} 3 \% \mathrm{~B} 4 \mathrm{te},+\mathrm{il}+\mathrm{ose}+$ lui + prendre $+\mathrm{la}+$ main. $+\mathrm{La}+$ jeun $\mathrm{e}+$ fille $+\mathrm{y}+\mathrm{est}+\mathrm{sensible} \% 22 \& \mathrm{hl}=\mathrm{pl} \& \mathrm{sa}=\mathrm{X} \& \mathrm{ved}=0$ ahUKEwi4uqD5yOLeAhXKWSwKHc- 1 DcoQ6AEIKzAA\#v=onepage \&q=\%22Tandis $\% 20$ qu $\%$ E2 $\% 80 \% 99$ ils $\% 20$ marchent $\% 20 \mathrm{c} \% \mathrm{C} 3$ $\%$ B4te $\% 20 \%$ C3\%A0\%20c\%C3\%B4te\%2C\%20il\%20ose\%20lui\%20prendre\%20la\%20main. $\% 20 \mathrm{La} \% 20$ jeune $\% 20$ fille $\% 20 \mathrm{y} \% 20$ est $\% 20$ sensible $\% 22 \& \mathrm{f}=$ false [20 XI 2018]

http://www.envoix.fr/chanter-jouer/27-quand-tu-marches-dans-mes-pas.html [20 XI 2018]

https://books.google.pl/books?id=4aQMAAAAYAAJ\&pg=PA37\&dq=\%22L $\% E 2 \% 80 \% 99$ import ation + des + esclaves + et + les + progr $\% \mathrm{C} 3 \% \mathrm{~A} 8 \mathrm{~s}+\mathrm{du}+$ christianisme + dans + la + population $+\% 22 \& \mathrm{hl}$

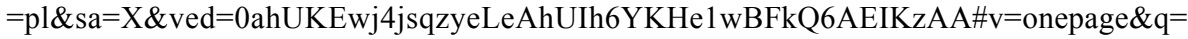
$\% 22 \mathrm{~L} \%$ E2\%80\%99importation\%20des\%20esclaves\%20et $\% 20$ les $\% 20$ progr $\% \mathrm{C} 3 \% \mathrm{~A} 8 \mathrm{~s} \% 20 \mathrm{du}$ $\% 20$ christianisme $\% 20$ dans\%201a\%20population\%20\%22\&f=false [20 XI 2018]

https://books.google.pl/books?id=-_9cAgAAQBAJ\&pg=PA47\&dq=\%22dirait+deux+amants+qui+ marchent $+\mathrm{c} \% \mathrm{C} 3 \% \mathrm{~B} 4 \mathrm{te}+\% \mathrm{C} 3 \% \mathrm{~A} 0+\mathrm{c} \% \mathrm{C} 3 \% \mathrm{~B} 4 \mathrm{te}+\mathrm{et}+\mathrm{la}+$ main + dans $+\mathrm{la}+$ main $\& \mathrm{hl}=\mathrm{pl} \& \mathrm{sa}=\mathrm{X} \&$ ved $=0$ ahUKEwj97qPnyeLeAhWGISwKHR0iDvsQ6AEIKTAA\# $\mathrm{v}=$ onepage $\& \mathrm{q}=\% 22$ dirait $\% 2$ 0deux $\% 20$ amants\%20qui $\% 20$ marchent $\% 20 \mathrm{c} \% \mathrm{C} 3 \% \mathrm{~B} 4$ te $\% 20 \% \mathrm{C} 3 \% \mathrm{~A} 0 \% 20 \mathrm{c} \% \mathrm{C} 3 \% \mathrm{~B} 4$ te $\% 20$ et $\% 201 \mathrm{a} \% 20$ main $\% 20$ dans $\% 201 \mathrm{a} \% 20$ main\&f=false [20 XI 2018]

http://chroniquesdemadoka.eklablog.com/saga-yakouba-jeunesse-beaux-livres-a133349492 [20 XI 2018]

https://www.lemonde.fr/sciences/article/2013/11/25/1-homme-nez-a-nez-avec-1-ours_3520007_ 1650684.html [20 XI 2018]

https://www.cnews.fr/france/2018-08-23/pyrenees-un-randonneur-se-retrouve-nez-nez-avec-uneourse-791913 [20 XI 2018]

https://www.lci.fr/insolite/video-nez-a-nez-avec-un-requin-blanc-2018118.html [20 XI 2018] https://cimtchau.ca/nouvelles/elle-tombe-face-face-avec-un-polaire/ [20 XI 2018]

https://www.touslesfestivals.com/actualites/visas-non-adaptes-accusations-infondees-pourquoi-lesartistes-se-font-ils-refouler-aux-frontieres-americaines-240417 [20 XI 2018]

https://gallica.bnf.fr/ark:/12148/bpt6k404353p/texteBrut [20 XI 2018]

https://www.notrepetitgraindasie.com/se-souvenir-des-belles-choses/ [20 XI 2018]

https://polar.zonelivre.fr/les-enquetes-de-nicolas-le-floch-en-bd/[20 XI 2018]

https://www.babelio.com/auteur/Yishai-Sarid/121030[20 XI 2018]

Exemples polonais notés Internet :

https://pl.wikisource.org/wiki/\%C5\%9Amier\%C4\%87_Felicyana_Dulskiego [25 XI 2018] https://books.google.pl/books?id=BqhODQAAQBAJ\&pg=PT53\&dq=\%22stan $\%$ C4\%85\%C5\%82 + nos + w + nos $+\mathrm{z} \% 22 \& \mathrm{hl}=$ pl\&sa $=$ X\&ved $=0$ ahUKEwiUrKSC5u_eAhXttYsKHUfxDkEQ6AEI $\mathrm{LjAB} \# \mathrm{v}=$ onepage $\& \mathrm{q}=\% 22 \operatorname{stan} \% \mathrm{C} 4 \% 85 \% \mathrm{C} 5 \% 82 \% 20$ nos $\% 20 \mathrm{w} \% 20$ nos $\% 20 \mathrm{z} \% 22 \& \mathrm{f}=$ false [25 XI 2018] 
https://syrenayachts.com/strona-glowna-2-2/targi/bavaria-cruiser-45/ [25 XI 2018]

https://film.wp.pl/jurassic-world-bryce-dallas-howard-nos-w-nos-z-dinozaurem-6022699015479937a

[25 XI 2018]

https://pl.wikisource.org/wiki/Strona:Bronis\%C5\%82aw_Malinowski_-_Wierzenia_pierwotne_i_ formy_ustroju_spo\%C5\%82ecznego.pdf/187 [25 XI 2018]

http://phrasebook_pl.enacademic.com/2303/oko [25 XI 2018]

https://books.google.pl/books?id=x0VRAgAAQBAJ\&pg=PA253\&dq=ajwa\%C5\%BCniejsze, $+\%$

$\mathrm{C} 5 \% \mathrm{BCe}+$ Annie $+\mathrm{si} \% \mathrm{C} 4 \% 99+\mathrm{uda} \% \mathrm{C} 5 \% 82 \mathrm{o}+\mathrm{Stan} \% \mathrm{C} 4 \% 99 \% \mathrm{C} 5 \% 82 \mathrm{a}+$ twarz $\% \mathrm{C} 4 \% 85+\mathrm{w}+\mathrm{t}$ warz $+\mathrm{z}+$ chorob $\% \mathrm{C} 4 \% 85,+$ zmierzy $\% \mathrm{C} 5 \% 82 \mathrm{a}+\mathrm{si} \% \mathrm{C} 4 \% 99+\mathrm{z}+\mathrm{b} \% \mathrm{C} 3 \% \mathrm{~B} 31 \mathrm{em} \& \mathrm{hl}=\mathrm{pl} \& \mathrm{sa}=\mathrm{X} \&$ ved=0ahUKEwi8pNO44-_eAhWupIsKHXLXAHcQ6AEILTAA\#v=onepage \&q=ajwa $\% \mathrm{C} 5 \%$ BCniejsze $\% 2 \mathrm{C} \% 20 \% \mathrm{C} 5 \% \mathrm{BCe} \% 20$ Annie $\% 20$ si $\% \mathrm{C} 4 \% 99 \% 20$ uda $\% \mathrm{C} 5 \% 820 . \% 20 \mathrm{Stan} \% \mathrm{C} 4 \%$ 99\%C5\%82a \%20twarz\%C4\%85\%20w\%20twarz $\% 20$ z $\% 20$ chorob\%C4\%85\%2C\%20zmierzy \%C5\%82a\%20si\%C4\%99\%20z\%20b\%C3\%B3lem\&f=false [25 XI 2018]

https://books.google.pl/books?id=nnHBCwAAQBAJ\&pg=PA18\&lpg=PA18\&dq=\%22stan $\% \mathrm{C} 4 \%$ $85 \% \mathrm{C} 5 \% 82+$ oko $+\mathrm{w}+$ oko $+\mathrm{ze} \% 22 \&$ source $=$ bl\&ots $=\mathrm{rY} 27 \mathrm{dP} 07 \mathrm{Lb} \&$ sig $=$ ELqFvgLI6LJER20Eu 6Ho8crJMpA\&hl=pl\&sa=X\&ved=2ahUKEwjumZmb8-_eAhWGkCwKHQwgA7wQ6AEw DHoECAIQAQ\#v=onepage\&q=\%22stan $\% \mathrm{C} 4 \% 85 \% \mathrm{C} 5 \% 82 \% 20 \mathrm{oko} \% 20 \mathrm{w} \% 20 \mathrm{oko} \% 20 \mathrm{ze} \% 2$ $2 \& \mathrm{f}=$ false [25 XI 2018]

http://bydgoszcz.wyborcza.pl/bydgoszcz/51,48722,11335172.html?i=1 [25 XI 2018]

https://books.google.pl/books?id=ALELCgAAQBAJ\&pg=PA57\&lpg=PA57\&dq=\%22szed\%C5\%

$82+\mathrm{r} \% \mathrm{C} 4 \% 99 \mathrm{ka}+\mathrm{w}+\mathrm{r} \% \mathrm{C} 4 \% 99 \mathrm{k} \% \mathrm{C} 4 \% 99 \% 22 \&$ source $=$ bl\&ots $=7 \mathrm{RQmAD} 55 \mathrm{Za} \&$ sig $=\mathrm{ulUaW}$

8c7rYqFrNSOSi4Motfewa4\&hl=pl\&sa=X\&ved=2ahUKEwiPxZbj4e_eAhWuiIsKHYxrBlEQ 6AEwDXoECAAQAQ\#v=onepage \&q=\%22 $\mathrm{szed} \% \mathrm{C} 5 \% 82 \% 20 \mathrm{r} \% \mathrm{C} 4 \% 99 \mathrm{ka} \% 20 \mathrm{w} \% 20 \mathrm{r} \% \mathrm{C} 4$ $\% 99 \mathrm{k} \% \mathrm{C} 4 \% 99 \% 22 \& \mathrm{f}=$ false [25 XI 2018]

https://www.telemagazyn.pl/artykuly/piecdziesiat-twarzy-blacka-czyli-parodia-bestselleru-eljamesw-kinach-zdjecia-wideo-47774.html [25 XI 2018]

https:/industrial.omron.pl/pl/services-support/events/indumation-belgium [25 XI 2018]

Exemples italiens notés Internet :

https://residentevilzone.forumfree.it/?t=73585053 [25 XI 2018]

https://books.google.pl/books?id=avcaAQAAIAAJ\&q=\%22naso $+a+$ naso + con $+\mathrm{il} \% 22 \& \mathrm{dq}=\% 22$ na

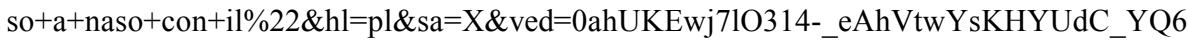
AEIRzAE [25 XI 2018]

http://www.gianfrancoiovino.it/MUSICA/QUANTO\%20AMORE\%20C\%27E\%27.html [25 XI 2018] https://books.google.pl/books?id=d-xLDwAAQBAJ\&pg=PT70\&dq=\%22faccia $+\mathrm{a}+$ faccia + con + la + tigre $\% 22 \& \mathrm{hl}=$ pl\&sa $=X \& v e d=0 a h U K E w j V 9 d D-w-\_$eAhUCx4sKHf4EAQQQ6AEIKTAA\#v= onepage $\& q=\% 22$ faccia $\% 20 \mathrm{a} \% 20$ faccia $\% 20$ con $\% 201 \mathrm{a} \% 20$ tigre $\% 22 \& \mathrm{f}=$ false [ 25 XI 2018] https://www.tripadvisor.it/ShowUserReviews-g190454-d1835077-r516993847-Haus_des_Meeres_

Aqua_Terra_Zoo-Vienna.html [25 XI 2018]

http://leggerefantastico.blogspot.com/2011/04/recensione-cappuccetto-rosso-sangue.html [25 XI 2018] https://www.lazialionair.it/2018/06/21/editoriale-di-danilo-galdino-giovedi-21-giugno-2018/ [25 XI

2018]

https://www.amazon.it/Cambio-pelle-Cesare-Veneziani/dp/8898838522 [25 XI 2018]

https://books.google.pl/books?id=0mhOAwAAQBAJ\&pg=PT35\&dq=\%22era + fianco $+\mathrm{a}+$ fianco +

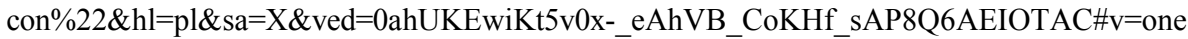
page \&q=\%22era $\% 20$ fianco $\% 20 \mathrm{a} \% 20$ fianco $\% 20$ con $\% 22 \& \mathrm{f}=$ false [25 XI 2018] 
https://books.google.pl/books?id=3KMCAwAAQBAJ\&pg=PT167\&dq=\%22si + ritrovano + mano+ nella + mano $\% 22 \& \mathrm{hl}=$ pl\&sa $=X \& v e d=0$ ahUKEwiem-_Z4u_eAhXpAhAIHXNYAkgQ6AEIM $\mathrm{TAB} \# \mathrm{v}=$ onepage $\& \mathrm{q}=\% 22 \mathrm{si} \% 20$ ritrovano $\% 20 \mathrm{mano} \% 20$ nella $\% 20 \mathrm{mano} \% 22 \& \mathrm{f}=$ false $[25 \mathrm{XI}$ 2018]

http://www.carriereinternazionali.com/cooperazione-sviluppo-e-volontariato/itemlist/user/11946federicasurace [25 XI 2018]

http://matematica.unibocconi.it/articoli/spiritualit\%C3\%A0-e-realt\%C3\%A0-una-visione-umanis tica-e-scientifica-del-mondo [25 XI 2018] 